\title{
Acción colectiva virtual y espacios de participación ciudadana de los jóvenes en Nicaragua ${ }^{1}$ \\ Virtual Collective Action and Spaces for Youth Participation in Nicaragua
}

\author{
Luis Jara ${ }^{2}$ \\ (iD https://orcid.org/0000-0001-9193-2804 \\ A.R. Saldierna ${ }^{3}$ \\ (iD https://orcid.org/0000-0003-1805-9740 \\ Universidad Autónoma de Nuevo León, México
}

Como referenciar este artículo:

Jara, L. \& Saldierna, A. R. (2020). Acción colectiva virtual y espacios de participación ciudadana de los jóvenes en Nicaragua. Revista Ciencia Jurídica y Política, 62-79. Recuperado de: $\quad$ https://portalderevistas.upoli.edu.ni/index.php/5revciencasjuridicasypoliticas/article/view/636

\section{RESUMEN}

El presente trabajo analiza la relación existente entre el uso de redes sociales y los niveles de participación ciudadana. En concreto, se explora si la participación ciudadana de los jóvenes en Nicaragua está relacionada con el uso de las diferentes redes sociales como mecanismo de interacción virtual y generación de espacios en la construcción de una consciencia crítica necesaria para el fortalecimiento de la Democracia. Para el estudio, se hace uso de la metodología cualitativa, teniendo como herramienta de recolección de datos la entrevista a profundidad. El muestreo estuvo acotado al proceso de interacción de los participantes, la riqueza de la información y la saturación de esta, conformando una muestra final de 10 entrevistas a líderes de movimientos universitarios en Nicaragua. El resultado de la investigación destaca la preferencia de los medios tecnológicos, aplicaciones (apps) y redes sociales virtuales para informar y compartir contenido relevante para la vida política y social, junto al desinterés del ciudadano joven a participar por medios convencionales en los asuntos públicos. Palabras claves: Acción colectiva virtual, jóvenes, participación ciudadana, redes sociales

\begin{abstract}
This work analyses the relationship between the social networks and the level of citizen participation. Specially, it explores if the civic participation of young people in Nicaragua is related to the uses of social network as a mechanism of virtual interaction and generation of spaces in the construction of a critical awareness necessary for the strengthening of democracy. For the study, is used a qualitative methodology, using the indepth interview as data collection tool. The sampling was limited to the process of interaction of the participants, the richness of the information and the saturation of it, forming a final sample of 10 interviewers with leaders of movements in Nicaragua. The result of the research highlights the preference of the technological means, applications (apps) and virtual social networks to inform and share relevant content to political and social life, along with the young citizen's disinterest in engaging in public affairs by conventional means.
\end{abstract}

Keywords: Virtual Collective Action, Citizen Participation, Social networks.

\footnotetext{
${ }^{1}$ Artículo derivado del proyecto de tesis "Movimientos Universitarios, Redes Sociales y Participación Ciudadana: Incidencia de la acción colectiva virtual en los espacios de participación ciudadana en Nicaragua".

${ }^{2}$ Maestrando en Ciencias Políticas por la Universidad Autónoma de Nuevo León. Becario CONACYT. Colaborador del Laboratorio de Comunicación Política. E-mail: luisjara.pacheco@gmail.com

${ }^{3}$ Dra. en Filosofía con Acentuación en Ciencias Políticas. Profesora titular de la Facultad de Ciencias Políticas y Relaciones Internacionales, Universidad Autónoma de Nuevo León. Investigador nacional nivel I y auxiliar administrativo del Laboratorio de Comunicación Política. E-mail: alma.saldiernasls@uanl.edu.mx
} 


\section{INTRODUCCIÓN}

Es innegable el antagonismo que existe entre la acción colectiva y el autoritarismo presente en algunas prácticas gubernamentales. A esta discrepancia se agrega la polarización, los extremismos y la radicalización que consienten el control de los medios de comunicación tradicionales y, a su vez, promueven la (re)conceptualización e interiorización forzosa de valores que responden únicamente a la materialización de intereses particulares, lo que genera "gran frustración y creciente movilización ciudadana alrededor del sistema-mundo" (Fuchs, 2012 en Valencia \& García, 2014, p. 21). Ante tal escenario, los jóvenes miembros de movimientos universitarios promueven la búsqueda de la concertación por medio de las redes de Internet y se infiere que la interacción entre gobernantes y gobernados en el espacio digital fomenta el compromiso político.

En ese sentido, el uso de las redes sociales virtuales comprende la amplificación del interés en lo político, así como, la organización y participación del ciudadano en los asuntos públicos. Sirva de ejemplo, las movilizaciones de estudiantes en Chile en 2011 y las de \#YoSoy132 en México en 2012 junto al uso de las redes sociales para informar a la comunidad y motivar la participación ciudadana. En 2018, tomando en cuenta la dinámica de las movilizaciones sociales en el sistema-mundo, movimientos de estudiantes universitarios en Nicaragua se auxilian de las bondades de las redes sociales.

Plataformas digitales como Facebook o Twitter y aplicaciones de mensajería (messaging apps) como WhatsApp son consideradas herramientas útiles para comunicar, compartir información y promover la participación del ciudadano joven. De ahí que la (hiper)información sea necesaria para construir una sociedad critica, construir nuevos espacios de participación y transformar la masa social.

En el caso de países como Nicaragua, la revisión bibliográfica expone que aún son exiguas las investigaciones científicas respecto a la incidencia de la acción colectiva en modalidad virtual en los espacios de participación ciudadana. En el país centroamericano existen investigaciones que abordan el tema de movimientos sociales y partidos políticos (Quezada \& Terán, 2005), movimientos sociales y su vinculación a grupos étnicos, mujeres y trabajadores (Sánchez, Castro, Rodríguez \& Guerra, 2016; Chaguaceda, 2011), así mismo, 
desde una perspectiva sociológica se analiza el fenómeno de la participación ciudadana y los movimientos sociales (Serra, 2003).

Lo anterior demuestra la carencia de investigaciones de vanguardia relacionadas al uso de las redes sociales en beneficio de la actividad de los movimientos universitarios y la incidencia de lo virtual en la participación ciudadana de los jóvenes nicaragüenses, sobre todo, en un contexto de tensión social. En particular, se constituye la "acción colectiva" como variable independiente que representa el estímulo, los inputs o las causas y, "participación ciudadana" como variable dependiente que representa el efecto. La variable dependiente también es conocida como variable de salida u output (Buendía, Cólas \& Hernández, 2001). El uso de las redes sociales se concibe como una variante moderadora de la relación entre las dos variables.

La importancia de analizar jóvenes radica en que de acuerdo a datos del Banco Central de Nicaragua (2017), más de la mitad de la población, considerada en 6.545.502 habitantes (Banco Mundial [BM], 2020), tiene menos de 24 años y se estima que el 60 por ciento de la población es menor de 30 años (Muñoz \& Narváez, 2018). Por tanto, la juventud resulta ser mayoría en Nicaragua y la participación de la mayoría es fundamental para el desarrollo de “sistemas y regímenes políticos donde el pueblo es el que manda" (Sartori, 2009, p. 15).

En ese sentido, el propósito de este trabajo de comunicación científica, es -dentro del contexto de los movimientos sociales universitarios- conocer el uso de las redes sociales en la construcción de espacios de acción colectiva y de activación de la participación para el ciudadano joven nicaragüense.

\section{MARCO TEÓRICO}

\section{Movimientos sociales y universitarios}

El cambio radical de factores y circunstancias incide en la ambigüedad o falta de unanimidad del término movimiento social, puesto que, distintas realidades se hacen notar ante la mirada de los estudiosos del tema (Laraña, 1996). Autores como Tilly y Wood (2010) construyen el concepto desde una visión historicista y se hace referencia a reivindicaciones del colectivo 
contra las autoridades en el poder. Tarrow (1997), por su parte, sostiene que los movimientos sociales son "desafíos colectivos planteados por personas que comparten objetivos comunes y solidaridad en una interacción mantenida por las élites, los oponentes y las autoridades" ( $\mathrm{p}$. 21). En general, se hace notar la activación de una conciencia que promueve actitudes y movilización. La naturaleza del movimiento social desafía el orden previamente establecido, a su vez, combate toda amenaza a los derechos económicos, políticos y sociales; en concreto amenazas presentes en las prácticas de gobiernos pseudodemocraticos y carentes de respeto al Estado de derecho. En efecto, "el movimiento social es un contrapeso a un poder opresivo, un llamamiento a la acción popular contra un amplísimo abanico de plagas" (Tilly \& Wodd, 2010, p. 20).

El estudio de los movimientos sociales denominados como "nuevos movimientos" tiene su antecedente en los años sesenta y su presencia en el sistema evidencia las “[...] contradicciones entre el individuo y el Estado: nuevos valores que cuestionan el estado de cosas, a partir de intereses sociales universales" (Aranda, 2000, p. 228). Entre los nuevos movimientos sociales destaca el movimiento de estudiantes universitarios.

En particular, el movimiento de estudiantes universitarios es un movimiento social que se renueva constantemente, dispone de una estructura organizacional que motiva a la movilización, utiliza estrategias como la resistencia pasiva, protestas o manifestaciones para lograr una transformación social y reducir la insatisfacción de la sociedad, sin embargo, debido a su carácter generacional presenta cierta estabilidad (Caycedo, 1984; Aranda, 2000). Generalmente el movimiento estudiantil está conformado por jóvenes entre 16 y 30 años, en su mayoría pertenecientes a la clase media. Así mismo, Zermeño (1978), citado por Aranda (2000, p. 242), agrega que "se trata de grupos pertenecientes a sectores modernos de la sociedad y la economía, es decir, relacionados con la ciencia y la tecnología y, en general, con la producción de conocimientos modernos".

En ese sentido, la producción académica y científica guarda estrecha relación con la construcción de lo común y es en el ámbito universitario donde se obtiene el conocimiento suficiente para iniciar discusiones que contribuyen al desarrollo de una sociedad más justa. La justicia es un principio conciliatorio, atenúa la ira, asegura la existencia y convivencia 
pacífica de la sociedad políticamente organizada. El conocimiento permite la formación de una sociedad crítica que impulsa el cambio, en concreto "el más auténtico amigo [...] del pueblo será el crítico frecuente, por no decir constante, del pueblo, y, aún más, el crítico de los aduladores políticos y culturales del pueblo o el crítico de la sabiduría popular" (Strauss, 2007, p. 17). El movimiento universitario, por tanto, resulta ser el vínculo entre la comunidad, la investigación y la discusión. La mayor reivindicación que tomar por la juventud organizada es de índole político y se intenta incidir en la conducción de la colectividad, para tal efecto, la crítica debe asociarse con la moral del movimiento y esta a su vez influir positivamente en la práctica política.

\section{Redes sociales y movilización ciudadana}

Estudios realizados demuestran que el uso de las redes sociales fomenta la participación política, no sólo en línea, sino también en el plano físico y esa participación se materializa en forma de manifestaciones, protestas, marchas, entre otras actividades colectivas (Park \& Kaye 2018; Gil de Zúñiga, Molyneux \& Zheng, 2014). Las virtudes de las redes sociales no se limitan al espacio digital y fomentan el compromiso político (Aguilera \& Casero-Ripollés, 2018). Sirva de ejemplo, las movilizaciones de estudiantes en Chile en 2011 y las de \#YoSoy132 en México en 2012 junto al uso de las redes sociales para informar a la comunidad y motivar la participación ciudadana.

El estudio concreto de los movimientos universitarios en Chile expone que, en el año 2011, los estudiantes realizaron manifestaciones en rechazo a la incorporación de inversionistas a la Universidad Central (alma mater que goza de autonomía institucional). En ese contexto, los estudiantes chilenos reconocen en la acción de las autoridades universitarias el interés de privatizar la educación superior, a su vez la situación expone los problemas del sistema de educación y las prácticas de mercado que priman sobre los intereses de la comunidad de estudiantes y docentes (Labraña, 2018). Por otro lado, en el caso de México, Valencia y García (2014) aseguran que la conformación del movimiento estudiantil \#YoSoy132, durante la campaña presidencial:

gestado gracias a la utilización de las redes sociales [...] estableció la plataforma política y de acción social que logro desestabilizar el control y la coacción que los 
poderes políticos ejercen conjuntamente con los medios masivos de comunicación; en concreto, la imposición mediática de un candidato presidencial (p. 184).

Así, la eficacia generada por el uso de tecnología permite la rápida difusión de iniciativas y el incremento de movilizaciones civiles (Espinar-Ruiz \& González-Río, 2015). En efecto, investigaciones científicas afirman que los movimientos antes citados han utilizado las redes sociales como instrumentos que permiten transformar la acción política a través de la interacción virtual, organización, movilización y participación del ciudadano en los asuntos públicos, generando así un impacto positivo en la realidad social (Sola-Morales; BacallaoPino, 2016; Cabalin; Valencia \& García, 2014).

Cabe señalar que los movimientos sociales estiman conveniente practicar la acción colectiva e invitar a la movilización ciudadana mediante la construcción de un proceso de comunicación independiente, es decir, libre de todo dominio (Castells, 2012) y esa conveniencia es ofertada por los medios de comunicación social en Internet.

\section{Participación política en un entorno virtual}

Resulta evidente, sin demostración alguna, que la participación del zoon politikón se reduce a la presencia física del individuo en una plaza pública o en una urna al depositar su consentimiento de quién o quiénes deben gobernar. El sujeto que adquiere derechos y obligaciones se convierte en un simple receptor de promesas políticas, por tanto, se excluye al ciudadano de la proposición de ideas o debates para la toma de decisiones. A contrario sensu, el ideal democrático contempla la participación del ciudadano en toda actividad que influye en las decisiones que orientan el sistema político (Yang \& DeHart, 2016), tomando en cuenta que la participación es el examen social que fortalece la confianza en el sistema, es un medio que mejora la lógica de la democracia (Navarro, 2004).

Es importante destacar, que en democracias de baja calidad, la conservación de mecanismos tradicionales de participación reduce el interés en la política, sin embargo, la inquietud social se aprovecha de las herramientas que brinda la tecnología para encontrar nuevas formas de informar, expresar, interaccionar con otros grupos y lograr el cumplimiento de objetivos

cívicos y políticos, formando así, para algunos teóricos, un compromiso político no 
constitucional o expresivo (Vaccari, et al., 2015; Keating \& Melis, 2017). Esto significa que, por medio de las redes sociales como Facebook, Twitter o Youtube, "las personas pueden iniciar de manera fácil [...] una petición sobre un tema político que es importante para ellos, y distribuirlo ampliamente no solo entre sus amigos, grupo, pero también mucho más allá” (Keating \& Melis, 2017, p. 879).

Del mismo modo, Keating y Melis (2017), afirman que "la revolución tecnológica provocada por Internet ha jugado un papel central en la proliferación de nuevos tipos de acción y expresión política” (p. 879). De ahí que Zumárraga, Reyes y Carofilis (2017), consideren que la participación en la red u online "hace referencia a actividades políticas que se distinguen de una participación fuera de la red $[\ldots]$ y que se realizan tanto en un entorno de Internet como en redes sociales" (p. 131). Como resultado, las tecnologías de información y comunicación han transformado la dinámica de la participación del ciudadano en los asuntos políticos.

Ciertamente, la participación online forma parte de la realidad social, mostrando un cambio en cuanto a la relación, mas no en la interacción entre individuos. La actividad política online fomenta distintas formas de participación y niveles de compromiso, no una reducción de estos (Sabariego \& Matos, 2018), sirva de ejemplo, la dimensión mediática y comunicacional del Movimiento 15-M en España y el poder de convocatoria de las prácticas audiovisuales del Movimiento 20 de febrero en Marruecos (Barbas, 2015; Benítez-Eyzaguirre, 2018), por mencionar algunos. Por tanto, la participación política online es una experiencia real y similar a toda participación convencional del ciudadano. La diferencia señala el contexto virtual en el que se interacciona (Yang \& DeHart, 2016).

\section{Jóvenes y política en Nicaragua}

Actualmente resulta tenue en la memoria del colectivo, el proceso de organización y concientización precedidos por movimientos de jóvenes estudiantes que contribuyen al cambio del sistema en defensa del interés nacional en Nicaragua. De acuerdo con Rocha (2018), para el revolucionario nicaragüense, Carlos Fonseca (1936-1976) los estudiantes universitarios no son apáticos a la política, el problema es su falta de activismo y sus métodos demasiado suaves. En realidad, en el país centroamericano "los jóvenes consideran que la 
etapa por la que atraviesan es principalmente para su preparación individual, la constitución de una base familiar propia y un tiempo de recreación” (Telléz, 2009, p. 32). A los jóvenes no les interesa conocer o conversar de política, así lo afirma el 54 por ciento de la población de jóvenes en la zona metropolitana de Managua, capital de Nicaragua (Mercado \& Vásquez, 2012).

Sin embargo, desde el 2018, jóvenes miembros de movimientos universitarios ponen de manifiesto los problemas sociopolíticos en el espacio virtual de las redes sociales. Se trata de una iniciativa que estimula la (re)activación del interés y participación del ciudadano en los asuntos que afectan a la comunidad políticamente organizada. Según Castro en Antunes, De Gori y Villacorta (2018), "la crisis política y social [...] en Nicaragua [...] resaltó la fuerte incidencia de las comunicaciones, en general, del Internet y las redes sociales, en particular, en la configuración del espacio político [...]” (p. 169). Como resultado, las plataformas digitales como Facebook y microblogs como Twitter son consideradas por los jóvenes universitarios como herramientas útiles para comunicar, compartir información y promover la participación del ciudadano y su interés en los asuntos políticos, en donde sin duda:

el uso de las tecnologías digitales e internet potencia las prácticas ciudadanas en la medida en que se estimula la comunicación entre pares, se accede a la información sin la intermediación de instituciones y se amplían las posibilidades de expresión de las personas (Mazo, 2011, p. 9).

En suma, el uso y trascendencia de las tecnologías de información y comunicación, permite conquistar el sentimiento mayoritario de los nicaragüenses. El civismo crece y la participación de los jóvenes en defensa y cumplimiento del pacto social los convierte en ciudadanos activos del proceso de concertación.

En ese sentido, la literatura revisada y las dinámicas sociales de los movimientos universitarios observadas, lleva a plantear las siguientes preguntas de investigación:

P1 ¿Cuál es el papel que tienen las redes sociales en la organización de los movimientos universitarios en Nicaragua? 
P2 ¿Cuál es la incidencia de las redes sociales en la construcción de espacios de participación ciudadana?

\section{MÉTODO}

Para la realización del estudio, se hizo uso de una metodología de corte cualitativo, consistente en la realización de entrevistas. Esto por ser una "herramienta eficaz para desentrañar significaciones, las cuales fueron elaboradas por los sujetos mediante sus discursos, relatos y experiencias" (Troncoso-Pantoja \& Amaya-Plascencia, 2017, p. 1), es un instrumento que permite captar la percepción y reconstruir el contexto desde los individuos (actores clave) que forman parte del sistema social.

\section{Muestra}

Es importante mencionar que la selección de los participantes estuvo limitada por la accesibilidad de algunos de ellos a querer participar en el estudio debido a la realidad que se vive en el contexto nicaragüense, sin embargo el abordaje desde una perspectiva académicacientífica sin fines de involucramiento político, permitió entrevistar a participantes plenamente identificados como integrantes de importantes movimientos universitarios en Nicaragua, como es la "Alianza Universitaria Nicaragüense" y el "Movimiento UNA 19 de Abril". Cabe mencionar que las edades de los participantes oscilaron entre los 18 y 29 años en concordancia con los rangos de edad del Código de la Niñez y la Adolescencia de Nicaragua y la Ley No. 392, Ley de Promoción del Desarrollo Integral de la Juventud; tales disposiciones legales consideran jóvenes a quienes se encuentran entre 18 y 29 años.

En ese sentido, el tamaño de la muestra estuvo determinado por un muestreo por "saturación", es decir que se estuvo entrevistando hasta que ya no se encontraron nuevos elementos que aportaran un conocimiento no existente o novedoso con respecto al tema analizado (Martínez-Salgado, 2017). Este tipo de muestreo permite tener calidad de los resultados y no cantidad de estos (Morse, 1995). 


\section{Proceso de aplicación del estudio:}

La recolección de la información tuvo lugar del 27 de abril al 25 de mayo de 2020, presentándose entre las limitaciones durante el proceso, la dificultad de contar con la participación de los entrevistados por las condiciones de pandemia a nivel internacional. Por ese motivo las entrevistas se realizaron en línea a través de la plataforma de Skype debido a su fácil acceso, calidad de sonido y seguridad. Cada entrevista fue grabada en video y audio previa autorización de los participantes para su posterior transcripción. La fase de transcripción fue realizada en semanas posteriores a la aplicación.

\section{Formulación del guion}

El diseño del guion contó con la elaboración de categorías deductivas previamente identificadas por los investigadores y que permitieran la construcción del fenómeno, esto a partir de supuestos teóricos que indicaban la relación entre el uso de las redes sociales y su efecto en la organización de movimientos sociales, dando como resultado las siguientes categorías.

Categoría 1: Núcleo personal: Se consideran los elementos que se relacionan al participante, como nivel de estudios, antecedentes de su familia, estudios, ideología, por ejemplo.

Categoría 2: Uso social de las tecnologías de información y comunicación (TIC's): donde se buscaba conocer el nivel de uso de esas redes, su confianza en este medio frente a los tradicionales.

Categoría 3: Acción colectiva en redes virtuales: en este apartado se buscó conocer la importancia de las redes sociales para el movimiento universitario, cómo los jóvenes confían en esos contenidos y cómo es el proceso de organización de la información y publicación de contenidos.

La validación del guion corrió a cargo de expertos, al considerar como "fuente de evidencia" el reconocimiento de la relevancia de las preguntas y su relación con el propósito del estudio de acuerdo a su experiencia en el tema (Escobar-Pérez \& Cuervo-Martínez, 2008). 


\section{ANÁLISIS DE RESULTADOS}

Del total de 10 jóvenes nicaragüenses entrevistados, la mayoría pertenecen al sexo femenino. Analizando el tipo de institución de educación superior a la que asisten la mayoría concurre a una universidad pública. Las edades de los jóvenes entrevistados oscilan entre los 18 y 29 años. Todos cuentan con una representación activa como líderes de los movimientos universitarios descritos en la muestra.

Observando cada categoría, es el Uso social de las tecnologías de información y comunicación, la que cumple la función de abstraer a los jóvenes líderes de movimientos universitarios en el tema, a fin de conocer el nivel de uso y confianza de las redes. En respuesta a esta categoría se hace notar un alto nivel de uso, no solo de redes sociales, si no también, de aplicaciones específicas para informarse, comunicarse y actuar, entre ellas destaca WhatsApp, Zoom y aplicaciones (apps) de prensa:

"Uso las redes sociales todos los días y todo el tiempo porque tengo la necesidad de informarme de lo que ocurre en mi país y en el mundo"

Participante, 18 años

"Para informarme uso todo el tiempo las redes sociales. También utilizo las aplicaciones de medios importantes como, por ejemplo, El País de España o el New York Times donde se puede acceder fácilmente a la información disponible"

Participante, 26 años

Es importante recalcar que ninguno de los jóvenes líderes entrevistados considera a los medios de comunicación tradicionales en Nicaragua como esenciales para informarse y comunicar. Tal actitud es preocupante porque demuestra un deterioro de la relación entre la función social de los medios de comunicación y la confianza del relevo generacional:

"Antes necesitábamos de los medios de comunicación tradicionales, por ejemplo, un espacio en un canal de televisión o radio, sin embargo, actualmente cada perfil, cada 
página en la red, puede convertirse en un transmisor de información, en un creador de actividades y convocatoria"

Participante, 25 años

En cuanto a la categoría de Acción colectiva en redes virtuales, que se concentra en conocer la importancia de las redes sociales en la actividad y proceso de los movimientos universitarios, se encontraron hallazgos relevantes.

En primer lugar, por consecuencia del clásico alejamiento entre gobernantes y gobernados, los jóvenes líderes que participan de la actividad de los movimientos universitarios califican como importante el uso de las redes sociales por que les permite conectarse y acercarse a un mayor número de ciudadanos, de manera fácil y rápida, esto en función de exponer sus demandas, exigir soluciones a los problemas que atañen la vida en sociedad e impactar en la opinión pública:

"Solo cuesta tener acceso a internet, lo cual es bastante cómodo porque es donde está la mayor concentración de población y esto hace que uno tenga la capacidad de llegar a la mayor cantidad de personas posibles"

Participante, 26 años

En segundo lugar, se estima que el contenido que se comparte desde el perfil social virtual de los movimientos universitarios se consume y genera un sentimiento de confianza en la juventud. Esto se debe a que los movimientos universitarios son percibidos como organizaciones ajenas a la política partidaria tradicional, lo que (re)afirma el desinterés del ciudadano joven a informarse por mass media tradicionales y participar por medios convencionales, por ejemplo, formar parte de partidos políticos, para aportar en la discusión y toma decisiones:

"Las personas que observan en línea las publicaciones de los movimientos universitarios sienten confianza porque reconocen que quienes comparten el mensaje son parte del relevo generacional. Ven a los jóvenes estudiantes como sujetos fuera de la 
dinámica de la política partidaria tradicional, lo que nos transforma en actores de responsabilidad y confianza"

Participante, 26 años

Tercero, con relación al proceso de organización de la información y publicación de contenidos, los movimientos universitarios establecen un área de comunicación compuesta por estudiantes universitarios de las carreras de comunicación social, diseño gráfico, entre otros. Además, los miembros de los movimientos universitarios, desde diferentes circunstancias territoriales en Nicaragua, envían la información pertinente. Es necesario recalcar que la propuesta digital se ve constantemente amenazada por el fenómeno de las fake news. No obstante, la práctica del networking y las alianzas cívicas con diferentes organizaciones funcionan como filtro para determinar la verdad o mentira de la información antes de compartir el producto comunicacional en la red.

\section{CONCLUSIONES}

El presente trabajo tuvo como objetivo analizar el papel que ejercen las redes sociales en la construcción de espacios de participación ciudadana y de organización en el contexto de los jóvenes nicaragüenses. Conocer el nivel de involucramiento y reconocimiento de los jóvenes como actores sociales es un problema previamente abordado, sobre todo, por los procesos democráticos a los que se ha enfrentado el país.

El por qué analizar a los jóvenes resulta decisivo por ser la generación que históricamente ha cuestionado las formas en que se estructura el poder político del Estado. En ese sentido, diversas discusiones intentan precisar esa categoría de jóvenes acotado a una generación. Así, por ejemplo, autores como Ortega y Gasset (1975) señalan que:

Lo decisivo en la idea de las generaciones no es que se sucedan, sino que se solapan o empalman. Siempre hay dos generaciones actuando al mismo tiempo, con plenitud de actuación, sobre los mismos temas y en torno a las mismas cosas, pero con distinto índice de edad, y por ello, con diverso sentido (p. 90). 
Esta forma de analizar a los jóvenes lleva a replantearse la forma en que se abordarán las experiencias, aspiraciones, pero también la perspectiva histórica en la que están inmersos y, cómo esto, los lleva a involucrarse en movimientos en búsqueda del cambio social. En ese sentido, sin duda los movimientos sociales han sido de gran relevancia para el sistema político como actores que influencian en la sociedad la constante búsqueda de respuesta a los conflictos. Autores como Della Porti y Dioni (2006) afirman que "parte de los elementos que favorecen su integración es que suponen una interacción informal, creencias compartidas, así como compromisos en la acción colectiva y protesta" (Della Porti \& Dioni en La Rosa, 2016, p, 51). Así mismo, la base de su estructura o el surgimiento de estos en la actualidad "están asociados a situaciones sociales previas coyunturales, actores sociales comprometidos y acceso a redes sociales" (La Rosa, 2016, p. 52).

En referencia al papel de las redes sociales en la configuración y organización de los movimientos sociales, este ha sido primordial. Ejemplos como la Primavera Árabe en 2010 y el Movimiento \#YoSoy132 en México en 2012, demuestran la relevancia del uso del recurso tecnológico intangible para organizarse. En gran medida, los problemas políticos, económicos y sociales, junto a la regularización de la naturaleza de los contenidos en los medios de comunicación tradicionales, han propiciado que los jóvenes se desencanten de las acciones de los decision makers y dirijan su interés a nuevos medios comunicación y plataformas de participación, en el caso de Nicaragua no es la excepción.

Precisamente, en el análisis de la relación del uso de las redes sociales en la generación de espacios de participación ciudadana, se puede encontrar en los jóvenes de Nicaragua, un dominante empleo de estas nuevas formas de comunicación, al declarar un elevado uso de redes como WhatsApp, Zoom y algunas aplicaciones (apps) de prensa, que les permiten contrastar la información, intercambiarla y comunicarse sin hacer uso de los medios tradicionales por su falta de confianza en lo que se presenta en televisión o prensa escrita local. Con eso estaríamos dando respuesta a la P1 que versaba ¿Cuál es el papel que tienen las redes sociales en la organización de los movimientos universitarios en Nicaragua?

En lo que respecta con la $\mathrm{P} 2$ ¿Cuál es la incidencia de las redes sociales en la construcción de espacios de participación ciudadana?, se encuentra que al no confiar en medios 
tradicionales realmente los jóvenes recurren a estas redes virtuales para informarse, debido a que conviven e intercambian comentarios e información con personas en las que confían y que perciben como veraz la información que ahí pueden encontrar, esto por considerar que tanto las personas que publican la información como el contenido mismo, está alejado al manejo político de un grupo en el poder contra el que luchan.

Además de la confianza en la información, el resultado de la investigación destaca la inmediatez de esta, así como, el alcance que tiene al llegar a una gran cantidad de población. En consecuencia, a pesar de la brecha tecnológica en el país, las plataformas virtuales permiten difundir información de fácil acceso, lo que promueve la formación de una ciudadanía crítica y favorece la lógica de la democracia.

En definitiva, las redes sociales se han convertido en una herramienta indispensable en la organización de movimientos universitarios en el contexto nicaragüense, al propiciar la acción colectiva y ser una canal y espacio de activación en lo que respecta a la participación ciudadana. Por tanto, el uso de redes sociales en Internet y aplicaciones es el primer paso para vencer la inactividad del ciudadano joven en la toma de decisiones.

\section{REFERENCIAS}

Aguilera, M. de, \& Casero-Ripollés, A. (2018). ¿Tecnologías para la transformación? Los medios sociales ante el cambio político y social. Revista ICONO14 Revista científica de Comunicación y Tecnologías emergentes, 16(1), 1-21.

Aranda, J. (2000). El Movimiento Estudiantil y la Teoría de los Movimientos Sociales. Convergencia. Revista de Ciencias Sociales, 7(21), 225-250.

Bacallao-Pino, L. (2016). Redes sociales, acción colectiva y elecciones: los usos de facebook por el movimiento estudiantil chileno durante la campaña electoral de 2013. Palabra Clave 19(3), 810-937.

Banco Central de Nicaragua. (2017). Nicaragua en cifras 2017. Consultado el 21 de marzo de https://www.bcn.gob.ni/publicaciones/periodicidad/anual/nicaragua_cifras

Banco Mundial. (2020, 3 de agosto). Nicaragua: Data source: United Nations World Population Prospects. Disponible en https://datos.bancomundial.org/pais/nicaragua

Barbas, Á. (2015). La comunicación educativa en el movimiento 15-M. Notas sobre una etnografía pedagógico-política en proceso. Kult-ur, 2(4). DOI: http://dx.doi.org/10.6035/Kult-ur.2015.2.4.9

Benítez-Eyzaguirre, L. (2018). Videoactivismo en Marruecos. El Movimiento 20 de Febrero. Revista Internacional de Pensamiento Político, 10, 119-134. Recuperado de https://www.upo.es/revistas/index.php/ripp/article/view/3589 
Buendía, L., Cólas, P., \& Hernández, F. (2001). Métodos de Investigación en Psicopedagogía. Madrid: McGraw-Hill.

Cabalin, C. (2014). Estudiantes conectados y movilizados: El uso de Facebook en las protestas estudiantiles en Chile. Revista Cientifica de Educomunicación, 22(43), 2533. DOI: http://doi.org/10.3916/C43-2014-02

Castro, I. (2018). Nicaragua: comunicación y redes en la crisis. En A. Antunes, E. De Gori \& C. Villacorta. (Eds). (2018). Nicaragua en crisis: Entre la revolución y la sublevación. Ciudad de Buenos Aires, Argentina: CLACSO. 159-176. Recuperado de http://www.jstor.org/stable/j.ctvnp0jft

Casanova, R. (2016). El claro oscuro en la historia del movimiento popular en Nicaragua. Recuperado de https://www.academia.edu/26223850/El_claro_oscuro_en_la_historia_del_movimie nto_popular_en_Nicaragua

Castells, M. (2012). Redes de indignación y esperanza. Madrid: Alianza Editorial.

Caycedo, J. (1984). Conceptos metodológicos para la historia del movimiento estudiantil colombiano. Revista Estudios Marxistas, 27, 48-60.

Chaguaceda, A. (2011). El movimiento de mujeres y las luchas sociales por la democratización en la Nicaragua postrevolucionaria (1990-2010). Encuentro, (89), 39-62.

Della Porta, D., \& Diani, M. (2006). Social Movements: An Introduction. Oxford: Blackwell. En La Rosa, A. (2016). Movimientos sociales, redes sociales y recursos simbólicos. Correspondencias y análisis, 6, 47-60.

Escobar-Pérez, J., \& Cuervo-Martínez, Á. (2008). Validez de contenido y juicio de expertos: una aproximación a su utilización. Avances en medición, 6(1), 27-36.

Espinar-Ruiz, E., \& González-Río, M. (2015). Uso de Internet y prácticas políticas de los jóvenes españoles. Convergencia, 22(69), 13-38.

Fuchs, C. (2012). Some reflections on Manuel Castells' book Networks of Outrage and Hope. Social Movements in the Internet Age. En Valencia J. \& García, C. (2014). Movimientos sociales e internet. Bogotá: Editorial Pontificia Universidad Javeriana.

Gil de Zuñiga, H., Molyneux, L., \& Zheng, P. (2014). Social Media, Political Expression, and Political Participation: Panel Analysis of Lagged and Concurrent Relationships. Journal of Communication, 64(4), 612-634. DOI: http//doi.org/10.1111/jcom.12103

Keating, A., \& Melis, G. (2017). Social media and youth political engagement: Preaching to the converted or providing a new voice for youth?. The British Journal of Politics and International Relations, 19(4), 877-894.

La Rosa, A. (2016). Movimientos sociales, redes sociales y recursos simbólicos. Correspondencias y análisis, 6, 47-60.

Labraña, J. (2018). La primavera chilena: no conservadora ni revolucionaria. Una explicación sociologica del significado historico del movimiento universitario chileno del año 2011. Calidad en la Educación, (48), 251-272.

Laraña, E. (1996). La actualidad de los clásicos y las teorías del comportamiento colectivo. Reis: Revista española de investigaciones sociológicas, 15-44.

Martínez-Salgado, C. (2012). El muestreo en investigación cualitativa: principios básicos y algunas controversias. Ciência \& Saúde Coletiva, 17(3), 613-619. DOI: https://dx.doi.org/10.1590/S1413-81232012000300006

Mazo, C. (2011). Clave para la información ciudadana en la era digital. Revista Q, 6(11), 114. 
Mercado, H., \& Vásquez, L. (2012). Cultura política de la juventud urbana de la región metropolitana de Managua. Encuentro, (91), 32-57.

Morse, J. (1995). The significance of saturation. Qualitative Health Research, 5(2), 147-149.

Muñoz, D., \& Narváez, M. (2018). ¿Cómo se informan y participan los jóvenes? Guía para organizaciones que trabajan con participación juvenil. Managua: FUNIDES.

Navarro, J. (2004, Noviembre). Participación ciudadana en la gestión parlamentaria: el concepto de "auditoría social". En Memorias del IX Congreso Internacional del CLAD sobre la Reforma del Estado y de la Administración Pública, 2(5), 1-13.

Ortega y Gasset (1975). Entorno a Galileo. Enciclopedia Internacional de las Ciencias Sociales. Madrid: Editorial Aguilar.

Park, C., \& Kaye, B. (2018). News Engagement on Social Media anda Democratic Citizenschip: Direct and Moderating Roles of Curatorial News Use In Political Involvement. Journalism \& Mass Communication Quarterly, 1-25.

Quezada, F., \& Terán, S. (2005). Partidos políticos y movimientos sociales en la Nicaragua de hoy. En La democracia y sus desafíos en Nicaragua, 83-125. CIELAC. Fundación Friedrich Ebert. Recuperado de http:// cielac-upoli/20120813015327/03partidos.pdf

Rocha, J. (2018). Las luchas universitarias en Nicaragua. Cómo se llegó a la masacre de estudiantes del 23 de julio de 1959. Envío digital, (438). Recuperado de https://www.envio.org.ni/articulo/5531

Sabariego, J., \& Matos, A. (2018). Entre a crise e a austeridade: potencialidades e desafios das novas Formas de ativismo dos recentes movimentos sociais globais em Espanha e Portugal. En II Congreso Internacional Move. net sobre Movimientos Sociales y TIC (2018), 373-385. Grupo Interdisciplinario de Estudios en Comunicación, Política y Cambio Social de la Universidad de Sevilla.

Sánchez, M., Castro, D., Rodríguez, R., \& Guerra, J. (2016). Movimientos sociales y acción colectiva en Nicaragua : entre la identidad, autonomía y subordinación. Amnis. Recuperado de http://journals.openedition.org/amnis/2813

Sartori, G. (2009). La Democracia en 30 Lecciones. México, D.F: Santillana Ediciones Generales.

Serra, L. (2003). Participación ciudadana y movimientos sociales. Encuentro, (64), 18-37. Recuperado de http://www.uca.edu.ni/2/images/Revista-Encuentro/Revistas/e64/art2.pdf

Sola-Morales, S. (2016). Las redes sociales y los nuevos movimientos estudiantiles latinoamericanos. La "Primavera Chilena" y el "\#YoSoy132". Revista Científica de Información y Comunicación (13), 153-193. Recuperado de https://ipena44.files.wordpress.com/2017/12/tripa-revista-ic-13-bn.pdf

Strauss, L. (2007). Liberalismo antiguo y moderno. Buenos Aires: Katz Editores.

Tarrow, S. (1997). El poder en movimiento. Los movimientos sociales, la acción colectiva y la política. Madrid: Alianza Editorial.

Telléz, D. (2009). La Exclusión Política de jóvenes, mujeres y pueblos indígenas: Propuestas para la Reforma Política en Nicaragua. Friedrich-Ebert-Stiftung.

Tilly, C., \& Wodd, J. (2010). Los Movimientos Sociales, 1768 - 2008: desde sus orígenes a Facebook. Barcelona: Editorial Crítica.

Troncoso-Pantoja, C., \& Amaya-Plascencia, A. ( 2017). Entrevista: guía práctica para la elaboración de datos cualitaivos en investigación en Salud. Rev. Fac. Med., 65(2), 329-332. DOI: https://doi.org/10.15446/revfacmed.v65n2.60235 
Vaccari, C., Valeriani, A., Barberá, P., Bonneau, R., Jost, J., Nagler, J., \& Tucker, J. (2015). Political expression and action on social media: Exploring the relationship between lower-and higher-threshold political activities among Twitter users in Italy. Journal of Computer-Mediated Communication, 20(2), 221-239.

Valencia, J., \& García, C. (2014). Movimientos sociales e internet. Bogotá: Editorial Pontificia Universidad Javeriana.

Yang, H., \& DeHart, J. (2016). Social Media Use and Online Political Participation Among College Students During the US Election 2012. Social Media + Society. DOI: https://doi.org/10.1177/2056305115623802

Zermeño, S. (1978). México una democracia utópica: El movimiento estudiantil del 68. México: Siglo XXI. En Aranda, J. (2000). El Movimiento Estudiantil y la Teoría de los Movimientos Sociales. Convergencia. Revista de Ciencias Sociales, 242.

Zumárraga, M., Reyes, C., \& Carofilis, C. (2017). ¿Verdad o ficción? El uso político de las redes sociales en la participación política offline en las elecciones presidenciales en ecuador. Análisis Político, $30(91)$, $130-145$.

DOI: https://doi.org/10.15446/anpol.v30n91.70268 